\title{
Central-moment description of polarization for quantum states of light
}

Björk, G.; Söderholm, J.; Kim, Y.-S.; Ra, Y.-S.; Lim, H.-T.; Kothe-Termén, Christian; Kim, Y.-H.; SánchezSoto, L. L.; Klimov, A. B.

Published in:

Physical Review A

Link to article, DOI:

10.1103/PhysRevA.85.053835

Publication date:

2012

Document Version

Peer reviewed version

Link back to DTU Orbit

Citation (APA):

Björk, G., Söderholm, J., Kim, Y-S., Ra, Y-S., Lim, H-T., Kothe-Termén, C., Kim, Y-H., Sánchez-Soto, L. L., \& Klimov, A. B. (2012). Central-moment description of polarization for quantum states of light. Physical Review $A$, 85, 053835. https://doi.org/10.1103/PhysRevA.85.053835

\section{General rights}

Copyright and moral rights for the publications made accessible in the public portal are retained by the authors and/or other copyright owners and it is a condition of accessing publications that users recognise and abide by the legal requirements associated with these rights.

- Users may download and print one copy of any publication from the public portal for the purpose of private study or research.

- You may not further distribute the material or use it for any profit-making activity or commercial gain

- You may freely distribute the URL identifying the publication in the public portal 


\title{
Central-moment description of polarization for quantum states of light
}

\author{
G. Björk, ${ }^{1}$ J. Söderholm, ${ }^{2}$ Y.-S. Kim, ${ }^{3}$ Y.-S. Ra, ${ }^{3}$ H.-T. Lim, ${ }^{3}$ C. Kothe, ${ }^{4}$ Y.-H. Kim, ${ }^{3}$ L. L. Sánchez-Soto, ${ }^{2,5}$ and A. B. Klimov ${ }^{6}$ \\ ${ }^{1}$ Department of Applied Physics, Royal Institute of Technology (KTH), AlbaNova University Center, SE-106 91 Stockholm, Sweden \\ ${ }^{2}$ Max Planck Institute for the Science of Light, Guenther-Scharowsky-Str. 1/Bldg. 24, 91058 Erlangen, Germany \\ ${ }^{3}$ Pohang University of Science and Technology (POSTECH), Pohang, 790-784, Korea \\ ${ }^{4}$ Department of Physics, Technical University of Denmark, 2800 Lyngby, Denmark \\ ${ }^{5}$ Departamento de Óptica, Facultad de Física, Universidad Complutense, 28040 Madrid, Spain \\ ${ }^{6}$ Departamento de Física, Universidad de Guadalajara, 44420 Guadalajara, Jalisco, Mexico
}

(Received 23 November 2011; published 25 May 2012)

\begin{abstract}
We present a moment expansion for the systematic characterization of the polarization properties of quantum states of light. Specifically, we link the method to the measurements of the Stokes operator in different directions on the Poincaré sphere and provide a scheme for polarization tomography without resorting to full-state tomography. We apply these ideas to the experimental first- and second-order polarization characterization of some two-photon quantum states. In addition, we show that there are classes of states whose polarization characteristics are dominated not by their first-order moments (i.e., the Stokes vector) but by higher-order polarization moments.
\end{abstract}

DOI: 10.1103/PhysRevA.85.053835

PACS number(s): 42.50.Ar, 42.25.Ja, 42.25.Kb

\section{INTRODUCTION}

A fundamental property of light is its vector nature. Far from a source, freely propagating light can be approximated by a plane wave, with the electric field lying in the plane perpendicular to the direction of propagation. The early pioneers of optics realized that a convenient way of characterizing light is to describe the figure the tip of the electric-field vector traces out in this plane. Stokes established an operational procedure to characterize not only the polarization properties of light but also to what extent a field is polarized [1]. The method is still the standard way of assessing polarization, although several generalizations, such as polarization of nonplane [2-5] and multimode [6-8] fields, have been developed. A limitation of Stokes' approach is that it only considers the average intensities (or photon numbers) and, hence, only assesses the first-order polarization moments.

As polarization is a relatively robust degree of freedom, that, moreover, can almost losslessly, cheaply, and easily be transformed, it is very often used for coding and manipulating quantum information. In free space, the polarization state is virtually isolated from the environment. In an optical fiber, environment induced birefringence may cause problems, but, typically, the changes are sufficiently slow to make them manageable. Examples of experiments relying on polarization include quantum key distribution $[9,10]$, quantum dense coding [11], quantum teleportation [12], quantum tomography [13], rotationally invariant states [14], phase super-resolution [15], and weak measurements [16]. However, many of these experiments use correlation measurements, effectively using second, or higher, polarization moments. Such correlation measurements can give surprising results. For example, states that appear unpolarized (that is, with vanishing Stokes parameters), can show unit-visibility polarization correlations when rotated on the Poincaré sphere [17]. Such states have been said to have "hidden polarization" [18,19]. As we shall discuss below, there are actually large classes of such states, and they can be classified by the number of lowest-order moments that are invariant under polarization transformations. We shall refer to such states as $r$ th-order unpolarized if the first $r$ moments are all invariant under any polarization rotation.

As hinted by this discussion, the full description of polarization can be sorted into moment orders and, simultaneously (but perhaps less obviously), into excitation manifolds. A convenient and experimentally palatable way to do this is by the use of central moments, so most of the paper will focus on these.

For the three lowest orders, the central moments coincide with the cumulants introduced by Thiele [20]. Each successive cumulant provides information of statistics not already contained in the lower-order cumulants. They have some advantages over a moment description when making affine transformations, and they also provide a simple method of quantifying the difference between a statistical distribution and its simplest Gaussian approximation [21]. (For Gaussian distributions all cumulants of order $\geqslant 3$ vanish.) Kubo promoted their use in quantum mechanics and thermodynamics [22] but in polarization optics they have been employed rather sparsely [23-27].

Below, we, first, recall some definitions and notation in Sec. II. In Secs. III and IV we examine how first- and second-order polarization properties can be described in terms of expectation values and central moments, respectively. In the following two sections, V and VI, we discuss how the centralmoment formalism can be extended to orders higher than the second. In Sec. VII we subsequently discuss the connection between excitation manifolds and polarization data and show that polarization tomography in general requires far less data than full-state tomography in agreement with Refs. [28-30]. We then apply the formalism, both theoretically and experimentally, to certain polarization states in Sec. VIII. In particular, we show that there are many states whose polarization characteristics are dominated not by their first-order moments (i.e., their Stokes vector) but by higher-order polarization moments. For example, for three-photon states there exist six different classes of states with different polarization characteristics. Finally, we draw some conclusions from the analysis in Sec. IX. 


\section{STOKES OPERATORS AND THE STOKES VECTOR}

We will build on the classical theory of polarization based on the Stokes parameters. For quantized fields, the Stokes operators [31] take the role of the Stokes parameters. They are

$$
\begin{array}{ll}
\hat{S}_{0}=\hat{a}_{H}^{\dagger} \hat{a}_{H}+\hat{a}_{V}^{\dagger} \hat{a}_{V}, & \hat{S}_{1}=\hat{a}_{H} \hat{a}_{V}^{\dagger}+\hat{a}_{H}^{\dagger} \hat{a}_{V}, \\
\hat{S}_{2}=i\left(\hat{a}_{H} \hat{a}_{V}^{\dagger}-\hat{a}_{H}^{\dagger} \hat{a}_{V}\right), & \hat{S}_{3}=\hat{a}_{H}^{\dagger} \hat{a}_{H}-\hat{a}_{V}^{\dagger} \hat{a}_{V},
\end{array}
$$

where $\hat{a}_{H}$ and $\hat{a}_{V}$ are the annihilation operators of the two orthogonal modes, in the following taken to be linearly horizontally and vertically oscillating electric fields, respectively. The annihilation operators obey the bosonic commutation relations

$$
\left[\hat{a}_{j}, \hat{a}_{k}^{\dagger}\right]=\delta_{j k}, \quad j, k \in\{H, V\} .
$$

The average values of the Stokes operators correspond to the Stokes parameters $\left(\left\langle\hat{S}_{0}\right\rangle,\langle\hat{\mathbf{S}}\rangle\right)$, where the Stokes vector operator is $\hat{\mathbf{S}}=\left(\hat{S}_{1}, \hat{S}_{2}, \hat{S}_{3}\right)$. In terms of the Poincaré sphere, the definitions (1) mean that $\hat{S}_{2}$ is the eigenoperator for a circularly polarized field and, thus, that the operator is "parallel" to the axis through the south (left-handed circular) and north pole (right-handed circular) of the sphere. $\hat{S}_{1}$ and $\hat{S}_{3}$ are the eigenoperators for diagonal and antidiagonal, and horizontal and vertical, linear polarization, respectively. These operators "lie" in the equatorial plane of the Poincaré sphere. The directions of $\hat{S}_{1}, \hat{S}_{2}$, and $\hat{S}_{3}$ form a right-handed orthogonal vector set in the Poincaré space.

As the Stokes operators differ from Schwinger's bosonic realization of the angular momentum operators [32] only by a factor 2 , the former satisfy the commutation relations of an su(2) algebra:

$$
\left[\hat{S}_{j}, \hat{S}_{k}\right]=i 2 \epsilon_{j k \ell} \hat{S}_{\ell},
$$

where $\epsilon_{j k \ell}$ is the Levi-Civita tensor. The noncommuting character of these operators leads to the uncertainty relation

$$
2\left\langle\hat{S}_{0}\right\rangle \leqslant\left\langle\hat{\mathbf{S}}^{2}\right\rangle-\langle\hat{\mathbf{S}}\rangle^{2} \leqslant\left\langle\hat{S}_{0}\right\rangle\left(\left\langle\hat{S}_{0}\right\rangle+2\right) .
$$

In spherical coordinates we can use the polar and azimuthal coordinates $\theta$ and $\phi$ to parametrize the unit vector as $\mathbf{n}=$ ( $\sin \theta \cos \phi, \sin \theta \sin \phi, \cos \theta$ ). (Note, however, that $\theta$ is the angle to $\hat{S}_{3}$, and that $\hat{S}_{1}$ and $\hat{S}_{3}$ lie in the equatorial plane of the Poincaré sphere, as explained above.) We can now express the Stokes operator in any direction $\mathbf{n}$ as

$$
\hat{S}_{\mathbf{n}}=\hat{\mathbf{S}} \cdot \mathbf{n}=n_{1} \hat{S}_{1}+n_{2} \hat{S}_{2}+n_{3} \hat{S}_{3}
$$

In addition to the commutation relation (3), one also has the relation

$$
\left[\hat{S}_{0}, \hat{S}_{j}\right]=0, \quad j \in\{1,2,3\} .
$$

This indicates that there exist simultaneous eigenstates of $\hat{S}_{0}$ (giving the total photon number) and any other Stokes operator. So, in principle, a measurement of $\hat{S}_{\mathbf{n}}$, if repeated on many members of an identically prepared ensemble, also allows the photon number statistics to be determined. In fact, a common way to measure any Stokes operator $\hat{S}_{\mathbf{n}}=\hat{U}_{\mathbf{n}} \hat{S}_{3} \hat{U}_{\mathbf{n}}^{\dagger}$, where $\hat{U}_{\mathbf{n}} \in \mathrm{SU}(2)$ is a (unitary) linear polarization transformation, is to first "rotate" the state according to $\hat{\varrho} \rightarrow \hat{U}_{\mathbf{n}}^{\dagger} \hat{\varrho} \hat{U}_{\mathbf{n}}$ and then measure $\hat{S}_{3}$. That is, after the rotation of the state, one separates the $H$ and $V$ modes by polarization optics and then counts the number of photons in each mode. The photocount difference then gives the measured $\hat{S}_{\mathbf{n}}$ eigenvalue, while the sum gives the $\hat{S}_{0}$ eigenvalue. This suggests that in a full description of quantum polarization, the excitation manifolds should be treated separately [28-30]. In consequence, coherences between different manifolds do not carry polarization information. Below, we shall use the total photon number $N$ as an index of the excitation manifold. As it will simplify the subsequent discussion, we introduce the normalized $N$-photon density matrix defined as

$$
\rho_{m n, N}=\frac{1}{p_{N}}\langle m, N-m|\hat{\rho}| n, N-n\rangle, \quad m, n \in\{0, \ldots, N\},
$$

where $p_{N}=\sum_{m=0}^{N}\langle m, N-m|\hat{\rho}| m, N-m\rangle$ (except for those $N$ where $p_{N}=0$ for which $\left.\rho_{m n, N}=0 \forall m, n \in\{0, \ldots, N\}\right)$. With this definition, we have

$$
\langle\hat{\mathbf{S}}\rangle_{N}=\operatorname{Tr}\left(\hat{\rho}_{N} \hat{\mathbf{S}}\right) .
$$

In reality, it may be experimentally difficult to divide the polarization measures into excitation manifolds, except for few-photon states. To this end, we shall also use the excitationaveraged Stokes vector

$$
\langle\hat{\mathbf{S}}\rangle=\sum_{N=1}^{\infty} p_{N}\langle\hat{\mathbf{S}}\rangle_{N}
$$

All other measures of polarization, defined below, can be averaged over the manifolds in the same manner. In Secs. VIII D and VIIIE this is done when discussing twomode coherent states and two-mode thermal states, states that when they are used often contain a large, but indeterminate, number of photons. However, for few-photon states it is possible to divide the results according to photon number through coincidence measurements, and we believe that it soon will become common to use detectors with photon number resolving capability for such states.

The idea that we will develop below is that the $r$ th-order polarization in the $N$ th excitation manifold is characterized by a data set that can predict $\left\langle\hat{S}_{\mathbf{n}}^{r}\right\rangle_{N}$ for any direction of the unit vector $\mathbf{n}$ on the Poincaré sphere.

\section{FIRST-ORDER POLARIZATION MOMENTS}

Since the classical description of polarization is based on the first-order moments, the quantum description is the direct translation of the classical description. That is, the Stokes vectors $\langle\hat{\mathbf{S}}\rangle_{N}$ defined in Eq. (8) give the complete first-moment polarization information. It follows from the expectation value of both sides of Eq. (5) with regards to the state $\hat{\rho}_{N}$ that $\langle\hat{\mathbf{S}}\rangle_{N}$ is sufficient to predict $\operatorname{Tr}\left(\hat{\rho}_{N} \hat{S}_{\mathbf{n}}\right)$ for any $\mathbf{n}$.

\section{ASSESSING THE SECOND-ORDER POLARIZATION MOMENTS}

How should one then go about to characterize higher-order polarization properties? One way would be to assess all second-order moments, i.e., all polarization correlation values of the form $T_{j k}^{(2, N)}(\hat{\rho})=\operatorname{Tr}\left(\hat{\rho}_{N} \hat{S}_{j} \hat{S}_{k}\right)$, where $j, k \in\{1,2,3\}$. 
However, these operator products are Hermitian only when $j=k$, so the expectation values cannot be measured directly. Nonetheless, from a theoretical perspective such an approach is viable and equivalent to the description via polarization moments in different directions. In Ref. [33] we have followed this route. A great simplification and reduction in data is to collect the polarization correlation information into Hermitian moment components [33]. One of this method's advantage is its simple hierarchy over the moment orders. It is straightforward to understand how to systematically collect the needed, nonredundant, information moment order by moment order. Experimentally, it is equivalent to the proposed method in that one measures successively higher moments of the Stokes operator for selected directions on the Poincaré sphere and then solves an ensuing equation system. A drawback is that it is not so easy to see the relative importance of the moment orders, as lower-order moments contribute to higher-order ones.

Another method is via the two-mode coherence matrices [19], where the $r$ th-order coherence matrix coefficient $j k$ is defined by $\left\langle\left(\hat{a}_{H}^{\dagger}\right)^{j}\left(\hat{a}_{V}^{\dagger}\right)^{r-j} \hat{a}_{H}^{k} \hat{a}_{V}^{r-k}\right\rangle$. If the excitation manifolds are treated separately, one obtains a method that is informationally equivalent to the one we shall develop. Among the advantages of the former method is that all moment coefficients are expectation values of normally ordered annihilation and creation operators, making calculations for coherent states particularly easy. Another one is that for $N$-photon states, all coherence matrices of order $r>N$ vanish. However, the method has only an indirect connection to the Stokes operators, and the ensuing matrices give little direct "feeling" for the polarization properties of the state, although they contain all the needed data. Experimentally, the offdiagonal coefficients $j \neq k$ of the coherence matrices are not straightforward to measure. In Ref. [34] a method using phase plates and projection onto an $r$ th-order "intensity" of one of the modes, namely $\left\langle\left(\hat{a}_{H}^{\dagger}\right)^{r} \hat{a}_{H}^{r}\right\rangle$, is proposed (note that this is not equivalent to a measurement of $\left.\left\langle\left(\hat{a}_{H}^{\dagger} \hat{a}_{H}\right)^{r}\right\rangle\right)$. Choosing properly $(r+1)^{2}$ different settings of the phase plates and solving the ensuing set of linear equations the coherence matrix of order $r$ can be obtained. For a state containing up to, and including, $N$ photons, $N\left(2 N^{2}+9 N+13\right) / 6$ measurements are, thus, required using the measurement scheme proposed in Ref. [34], roughly twice as many as for the scheme presented here; see the end of Sec. VI and Sec. VII below. The proposal in Ref. [34] discusses only characterization of $N$-photon states and not how to assess the polarization or higher-order coherence properties of states with an indeterminate number of photons.

To see how the polarization central moments appear quite naturally in a polarization description, we expand each operator in a state-dependent mean and a fluctuation part, viz.

$$
\hat{\Delta}_{\mathbf{n}, N}(\hat{\varrho}) \equiv \hat{S}_{\mathbf{n}}-\operatorname{Tr}\left(\hat{\varrho}_{N} \hat{S}_{\mathbf{n}}\right) .
$$

In the following, to simplify the notation, we shall write $\operatorname{Tr}\left(\hat{\varrho}_{N} \hat{S}_{\mathbf{n}}^{r}\right) \equiv\left\langle\hat{S}_{\mathbf{n}}^{r}\right\rangle_{N}$ and $\operatorname{Tr}\left[\hat{\varrho}_{N} \hat{\Delta}_{\mathbf{n}, N}^{r}(\hat{\varrho})\right] \equiv\left\langle\hat{\Delta}_{\mathbf{n}}^{r}\right\rangle_{N}$. This allows us to write, for $r=2$,

$$
\begin{aligned}
\left\langle\hat{S}_{\mathbf{n}}^{2}\right\rangle_{N}= & n_{1}^{2}\left(\left\langle\hat{S}_{1}\right\rangle_{N}^{2}+\left\langle\hat{\Delta}_{1}^{2}\right\rangle_{N}\right)+\text { c.p. }+ \text { c.p. } \\
& +n_{1} n_{2}\left(2\left\langle\hat{S}_{1}\right\rangle_{N}\left\langle\hat{S}_{2}\right\rangle_{N}+\left\langle\hat{\Delta}_{1} \hat{\Delta}_{2}\right\rangle_{N}+\left\langle\hat{\Delta}_{2} \hat{\Delta}_{1}\right\rangle_{N}\right) \\
& + \text { c.p. }+ \text { c.p. }
\end{aligned}
$$

where c.p. denotes a cyclic permutation of the indices. We see that apart from $\left\langle\hat{S}_{j}\right\rangle_{N}, j \in\{1,2,3\}$, the expectation values of the six Hermitian fluctuation "operators" in Eq. (11) are required to know $\left\langle\hat{S}_{\mathbf{n}}^{2}\right\rangle_{N}$ in any direction. These expectation values are the second-order central moments (coinciding with the second-order cumulant), defined as

$$
\left\langle\hat{\Delta}_{j} \hat{\Delta}_{k}\right\rangle_{N}=\left\langle\hat{S}_{j} \hat{S}_{k}\right\rangle_{N}-\left\langle\hat{S}_{j}\right\rangle_{N}\left\langle\hat{S}_{k}\right\rangle_{N}
$$

As can be seen from Eq. (11), it is convenient and natural to collect the mixed-product $(j \neq k)$ central moments into Hermitian terms, e.g., $\left\langle\hat{\Delta}_{j} \hat{\Delta}_{k}+\hat{\Delta}_{k} \hat{\Delta}_{j}\right\rangle_{N}$. These terms can be measured, and we see that, in addition to the Stokes parameters, we need six more numbers to fully characterize the secondorder polarization properties. The first three can be obtained from measuring the statistics of the Stokes vector $\hat{\mathbf{S}}$ yielding the first-order moments $\left\langle\left(\hat{S}_{1}, \hat{S}_{2}, \hat{S}_{3}\right)\right\rangle_{N}$ and the variances $\left\langle\hat{\Delta}_{j}^{2}\right\rangle_{N}$, $j \in\{1,2,3\}$. The additional three numbers can be obtained from measuring the statistics of $\hat{S}_{\mathbf{n}}$ along the "diagonal" directions $(1,1,0) / \sqrt{2},(1,0,1) / \sqrt{2}$, and $(0,1,1) / \sqrt{2}$ in the $\hat{S}_{1} \hat{S}_{2}, \hat{S}_{1} \hat{S}_{3}$, and $\hat{S}_{2} \hat{S}_{3}$ planes, respectively, corresponding to the angles $(\theta, \phi)$ of $(\pi / 2, \pi / 4),(\pi / 4,0)$, and $(\pi / 4, \pi / 2)$ on the Poincaré sphere and then using Eq. (11).

As a minor digression, these second-order central-moment terms are directly connected to the Hermitian polarization covariance matrix $\boldsymbol{\Gamma}_{N}$ with matrix coefficients

$$
\Gamma_{j k, N}=\frac{1}{2}\left\langle\hat{\Delta}_{j} \hat{\Delta}_{k}+\hat{\Delta}_{k} \hat{\Delta}_{j}\right\rangle_{N}
$$

where $j, k \in\{1,2,3\}$ [35]. Each such matrix has six independent coefficients as $\Gamma_{j k, N}=\Gamma_{k j, N}$ by construction. It is clear from Eq. (11) that this covariance matrix contains the information we need, in addition to the expectation value of the Stokes vector, to be able to predict the value of $\left\langle\hat{S}_{\mathbf{n}}^{2}\right\rangle_{N}$ in any direction. We also have $\left\langle\hat{\Delta}_{\mathbf{n}}^{2}\right\rangle_{N}=\mathbf{n} \cdot \boldsymbol{\Gamma}_{N} \cdot \mathbf{n}^{t}$, where $t$ denotes the transpose.

Every covariance matrix $\Gamma_{N}$ can be made diagonal by an orthogonal matrix. In this rotated, orthogonal coordinate system, where $\hat{S}_{\mathbf{e}_{j}}$ points in the direction of the eigenvector $\mathbf{e}_{j}$, of $\boldsymbol{\Gamma}_{N}$, one finds the extreme values of $\left\langle\hat{\Delta}_{\mathbf{n}}^{2}\right\rangle_{N}$. In this coordinate system, Eq. (11) simplifies to

$$
\left\langle\hat{\Delta}_{\mathbf{n}}^{2}\right\rangle_{N}=\lambda_{1}\left(\sin \theta^{\prime} \cos \phi^{\prime}\right)^{2}+\lambda_{2}\left(\sin \theta^{\prime} \sin \phi^{\prime}\right)^{2}+\lambda_{3} \cos ^{2} \theta^{\prime},
$$

where $\lambda_{j}$ is the eigenvalue of $\boldsymbol{\Gamma}_{N}$ associated with $\mathbf{e}_{j}, \theta^{\prime}$ is the angle between $\mathbf{n}$ and $\mathbf{e}_{3}$, and $\phi^{\prime}$ is the azimuthal angle in the $\mathbf{e}_{1}-\mathbf{e}_{2}$ plane. This equation may look like the equation of an ellipsoid, but it is not, as, e.g., one of the eigenvalues can can vanish, as is the case in Fig. 2, below.

In order to measure $\boldsymbol{\Gamma}_{N}$, one makes the same measurements as discussed above. The matrix $\Gamma_{N}$ can subsequently be deduced by solving Eq. (11) for $\left\langle\hat{S}_{j}\right\rangle_{N}$ and $\left\langle\hat{\Delta}_{j}^{2}\right\rangle_{N}$ given the measured values of $\left\langle\hat{S}_{\mathbf{n}}^{2}\right\rangle_{N}$ along the six directions. For better "immunity" to systematic errors, one could make measurements along additional directions and make a best fit of the ensuing overcomplete system of equations.

\section{THIRD-ORDER POLARIZATION}

Moving on to third-order moments, things get a bit more involved. Still, our underlying idea is that if one has all the 
central moments up to order 3 , then one can predict $\left\langle\hat{S}_{\mathbf{n}}^{3}\right\rangle_{N}$ for any direction.

We, therefore, first express the expectation value $\left\langle\hat{S}_{\mathbf{n}}\right\rangle_{N}^{3}$ in terms of $\langle\hat{\mathbf{S}}\rangle_{N}$,

$$
\begin{aligned}
\left\langle\hat{S}_{\mathbf{n}}\right\rangle_{N}^{3}= & n_{1}^{3}\left\langle\hat{S}_{1}\right\rangle_{N}^{3}+n_{2}^{3}\left\langle\hat{S}_{2}\right\rangle_{N}^{3}+n_{3}^{3}\left\langle\hat{S}_{3}\right\rangle_{N}^{3} \\
& +3\left(n_{1}^{2} n_{2}\left\langle\hat{S}_{1}\right\rangle_{N}^{2}\left\langle\hat{S}_{2}\right\rangle_{N}+n_{1}^{2} n_{3}\left\langle\hat{S}_{1}\right\rangle_{N}^{2}\left\langle\hat{S}_{3}\right\rangle_{N}\right. \\
& +n_{2}^{2} n_{1}\left\langle\hat{S}_{2}\right\rangle_{N}^{2}\left\langle\hat{S}_{1}\right\rangle_{N}+n_{2}^{2} n_{3}\left\langle\hat{S}_{2}\right\rangle_{N}^{2}\left\langle\hat{S}_{3}\right\rangle_{N} \\
& \left.+n_{3}^{2} n_{1}\left\langle\hat{S}_{3}\right\rangle_{N}^{2}\left\langle\hat{S}_{1}\right\rangle_{N}+n_{3}^{2} n_{2}\left\langle\hat{S}_{3}\right\rangle_{N}^{2}\left\langle\hat{S}_{2}\right\rangle_{N}\right) \\
& +6 n_{1} n_{2} n_{3}\left\langle\hat{S}_{1}\right\rangle_{N}\left\langle\hat{S}_{2}\right\rangle_{N}\left\langle\hat{S}_{3}\right\rangle_{N} .
\end{aligned}
$$

In a similar manner we can express the third-order raw moment of $\hat{S}_{\mathbf{n}}$ as

$$
\begin{aligned}
\left\langle\hat{S}_{\mathbf{n}}^{3}\right\rangle_{N}= & n_{1}^{3}\left(\left\langle\hat{\Delta}_{1}^{3}\right\rangle_{N}+3\left\langle\hat{S}_{1}\right\rangle_{N}\left\langle\hat{\Delta}_{1}^{2}\right\rangle_{N}\right) \\
& + \text { c.p. }+ \text { c.p. } \\
& +n_{1}^{2} n_{2}\left(3\left\langle\hat{S}_{1}\right\rangle_{N}\left\langle\hat{\Delta}_{1} \hat{\Delta}_{2}+\hat{\Delta}_{2} \hat{\Delta}_{1}\right\rangle_{N}\right. \\
& +3\left\langle\hat{S}_{2}\right\rangle_{N}\left\langle\hat{\Delta}_{1}^{2}\right\rangle_{N}+\left\langle\hat{\Delta}_{1}^{2} \hat{\Delta}_{2}+\hat{\Delta}_{2} \hat{\Delta}_{1}^{2}\right\rangle_{N} \\
& \left.+\left\langle\hat{\Delta}_{1} \hat{\Delta}_{2} \hat{\Delta}_{1}\right\rangle_{N}\right)+ \text { c.p. }+ \text { c.p. } \\
& +n_{1}^{2} n_{3}\left(3\left\langle\hat{S}_{1}\right\rangle_{N}\left\langle\hat{\Delta}_{1} \hat{\Delta}_{3}+\hat{\Delta}_{3} \hat{\Delta}_{1}\right\rangle_{N}\right. \\
& +3\left\langle\hat{S}_{3}\right\rangle_{N}\left\langle\hat{\Delta}_{1}^{2}\right\rangle_{N}+\left\langle\hat{\Delta}_{1}^{2} \hat{\Delta}_{3}+\hat{\Delta}_{3} \hat{\Delta}_{1}^{2}\right\rangle_{N} \\
& \left.+\left\langle\hat{\Delta}_{1} \hat{\Delta}_{3} \hat{\Delta}_{1}\right\rangle_{N}\right)+ \text { c.p. }+ \text { c.p. } \\
& +n_{1} n_{2} n_{3}\left(3\left\langle\hat{S}_{1}\right\rangle_{N}\left\langle\hat{\Delta}_{2} \hat{\Delta}_{3}+\hat{\Delta}_{3} \hat{\Delta}_{2}\right\rangle_{N}\right. \\
& +\left\langle\hat{\Delta}_{1} \hat{\Delta}_{2} \hat{\Delta}_{3}+\hat{\Delta}_{1} \hat{\Delta}_{3} \hat{\Delta}_{2}\right\rangle_{N} \\
& \left.+ \text { c.p. }+ \text { c.p. }+\left\langle\hat{S}_{\mathbf{n}}\right\rangle_{N}^{3}\right) .
\end{aligned}
$$

Finally, we can express the third-order central moments as

$$
\begin{aligned}
\left\langle\hat{\Delta}_{j} \hat{\Delta}_{k} \hat{\Delta}_{\ell}\right\rangle_{N}= & \left\langle\hat{S}_{j} \hat{S}_{k} \hat{S}_{\ell}\right\rangle_{N}-\left\langle\hat{S}_{j}\right\rangle_{N}\left\langle\hat{S}_{k} \hat{S}_{\ell}\right\rangle_{N} \\
& -\left\langle\hat{S}_{k}\right\rangle_{N}\left\langle\hat{S}_{j} \hat{S}_{\ell}\right\rangle_{N}-\left\langle\hat{S}_{\ell}\right\rangle_{N}\left\langle\hat{S}_{j} \hat{S}_{k}\right\rangle_{N} \\
& +2\left\langle\hat{S}_{j}\right\rangle_{N}\left\langle\hat{S}_{k}\right\rangle_{N}\left\langle\hat{S}_{\ell}\right\rangle_{N} .
\end{aligned}
$$

Hence, for the first to third orders, the central moments coincide with the cumulants.

One sees that in Eq. (16), if the 10 Hermitian, third-order, central-moment terms (each associated with a different geometric term $n_{j} n_{k} n_{3-j-k}$, where $j, k \in\{1,2,3\}$ and $j+k \leqslant 3$ ) are determined, in addition to the first- and second-order properties, then the third-order polarization properties are also determined for any direction. Hence, what one must measure are the sums of all fluctuation terms having $j$ ones, $k$ twos, and $3-j-k$ threes, where $j+k \leqslant 3$, or, more generally, for polarization order $r$, into sums having $r-j-k$ threes, where $j+k \leqslant r$.

Measuring the third-order fluctuations along, e.g., the $(\theta, \phi)$ directions $(0,0),(\pi / 2,0),(\pi / 2, \pi / 2),\left(\pi / 2, \phi_{1}\right),(\pi / 2$, $\left.-\phi_{1}\right),\left(\pi / 2-\phi_{1}, 0\right),\left(\pi / 2+\phi_{1}, 0\right),\left(\pi / 2-\phi_{1}, \pi / 2\right),(\pi / 2+$ $\left.\phi_{1}, \pi / 2\right)$, and $\left(\pi / 2-\phi_{1}, \pi / 4\right)$, where $\phi_{1}=\arccos \sqrt{2 / 3}$, one gets a system of 10 linearly independent equations that allows one to determine the terms $\left\langle\hat{\Delta}_{1}^{3}\right\rangle_{N}+3\left\langle\hat{S}_{1}\right\rangle_{N}\left\langle\hat{\Delta}_{1}^{2}\right\rangle_{N}$, and so on. Using the knowledge about the lower-order polarization terms, one can estimate the third-order terms, in this case $\left\langle\hat{\Delta}_{1}^{3}\right\rangle_{N}$. We note that the three first measurement directions are simply along the $\hat{S}_{1}, \hat{S}_{2}$, and $\hat{S}_{3}$ axes, so, in fact, only measurements along seven extra directions are required, in addition to the measurements along six directions needed to determine $\langle\hat{\mathbf{S}}\rangle_{N}$ and $\left\langle\hat{S}_{\mathbf{n}}^{2}\right\rangle_{N}$. Alternatively, if one wants to minimize the number of measurement directions, one can use the statistics collected when measuring along the six directions that determine the first- and second-order polarization moments and supplement them with measurements along the four new directions $(\pi / 6, \pi / 6),(\pi / 6, \pi / 3),(\pi / 3, \pi / 6)$, and $(\pi / 3, \pi / 3)$.

For third-order polarization the first thing to be considered is that the fluctuations of $\hat{S}_{\mathbf{n}}^{3}$ involves not only third powers of $\hat{\Delta}_{j}$ but also terms like $n_{1}^{3}\left\langle\hat{S}_{1}\right\rangle\left\langle\hat{\Delta}_{1}^{2}\right\rangle_{N}$ and $n_{1}^{2} n_{2}\left\langle\hat{S}_{1}\right\rangle_{N}\left\langle\hat{\Delta}_{1} \hat{\Delta}_{2}+\right.$ $\left.\hat{\Delta}_{2} \hat{\Delta}_{1}\right\rangle_{N}$. That is, the second- and third-order fluctuations become "intermixed" unless the state has vanishing Stokes parameters. This is in contrast to the (simpler) second order. A consequence of this is that if the state's first-order polarization is much larger than the square root of its variance, then all higher-order fluctuations will, in general, be dominated by the beating terms between the mean polarization vector and the second-order fluctuations. Hence, for most "reasonably excited" and "somewhat first-order polarized" states one does not have to go beyond the second-order moments to characterize the polarization fluctuations of all orders to a very good precision. However, for states having a small or vanishing first-order polarization, and for, e.g., the eigenstates to the Stokes operators in the direction of $\boldsymbol{\Gamma}_{N}$ 's eigenvector directions on the Poincare sphere, the polarization structures of orders higher than 2 will be of interest.

The expansions (11) and (16) also indicate an experimental advantage in describing the polarization in terms of its central moments. For each successive order it becomes quite clear to which accuracy one needs to measure the moments to obtain information not already contained in lower moments and, similarly, to what extent the higher-order central moments contribute to the raw moments. This information is, of course, implicit in "equivalent" descriptions such as generalized coherence matrices [19] or polarization tensors [33], but it is not explicitly displayed.

\section{FOURTH- AND HIGHER-ORDER POLARIZATION}

From the preceding sections it is rather clear how one could continue through the higher orders. In order to know $\left\langle\hat{S}_{\mathbf{n}}^{r}\right\rangle_{N}$ in any direction, the full set of Hermitian central-moment terms for all orders $\leqslant r$ is required.

Explicitly, the fourth-order central moments are

$$
\begin{aligned}
&\left\langle\hat{\Delta}_{j} \hat{\Delta}_{k} \hat{\Delta}_{\ell} \hat{\Delta}_{m}\right\rangle_{N} \\
&=\left\langle\hat{S}_{j} \hat{S}_{k} \hat{S}_{\ell} \hat{S}_{m}\right\rangle_{N}-\left\langle\hat{S}_{j}\right\rangle_{N}\left\langle\hat{S}_{k} \hat{S}_{\ell} \hat{S}_{m}\right\rangle_{N} \\
& \quad-\left\langle\hat{S}_{k}\right\rangle_{N}\left\langle\hat{S}_{j} \hat{S}_{\ell} \hat{S}_{m}\right\rangle_{N}-\left\langle\hat{S}_{\ell}\right\rangle_{N}\left\langle\hat{S}_{j} \hat{S}_{k} \hat{S}_{m}\right\rangle_{N} \\
& \quad-\left\langle\hat{S}_{m}\right\rangle_{N}\left\langle\hat{S}_{j} \hat{S}_{k} \hat{S}_{\ell}\right\rangle_{N}+\left\langle\hat{S}_{j}\right\rangle_{N}\left\langle\hat{S}_{k}\right\rangle_{N}\left\langle\hat{S}_{\ell} \hat{S}_{m}\right\rangle_{N} \\
& \quad+\left\langle\hat{S}_{j}\right\rangle_{N}\left\langle\hat{S}_{\ell}\right\rangle_{N}\left\langle\hat{S}_{k} \hat{S}_{m}\right\rangle_{N}+\left\langle\hat{S}_{j}\right\rangle_{N}\left\langle\hat{S}_{m}\right\rangle_{N}\left\langle\hat{S}_{k} \hat{S}_{\ell}\right\rangle_{N} \\
&+\left\langle\hat{S}_{k}\right\rangle_{N}\left\langle\hat{S}_{\ell}\right\rangle_{N}\left\langle\hat{S}_{j} \hat{S}_{m}\right\rangle_{N}+\left\langle\hat{S}_{k}\right\rangle_{N}\left\langle\hat{S}_{m}\right\rangle_{N}\left\langle\hat{S}_{j} \hat{S}_{\ell}\right\rangle_{N} \\
&+\left\langle\hat{S}_{\ell}\right\rangle_{N}\left\langle\hat{S}_{m}\right\rangle_{N}\left\langle\hat{S}_{j} \hat{S}_{k}\right\rangle_{N}-3\left\langle\hat{S}_{j}\right\rangle_{N}\left\langle\hat{S}_{k}\right\rangle_{N}\left\langle\hat{S}_{\ell}\right\rangle_{N}\left\langle\hat{S}_{m}\right\rangle_{N} .
\end{aligned}
$$

In contrast to the three lower orders, this result is not identical to the fourth-order cumulant. Higher-order central moments, which we will not write out explicitly, do not coincide with the cumulants either. 
Analogously with the second and third orders, we need not determine each term of the form (18) but only the Hermitian sum of moments associated to a certain geometrical prefactor. The number of such central-moment sum terms specific to the order $r$ is $(r+1)(r+2) / 2$ and the complete set of such terms up to, and including, order $r$ is $r\left(r^{2}+6 r+11\right) / 6$. To obtain the terms, one would have to measure the polarization statistics along such a number of carefully selected directions, yielding a complete, "maximally" linearly independent set of equations that could be solved numerically. To obtain better accuracy one could "oversample" the polarization statistics over the Poincaré sphere and use maximum likelihood or entropy methods to make a better estimate. However, as the states that have their polarization characteristics mainly determined by the $r$ th-order moment will be rather elaborated as $r$ increases, we expect the interest in the polarization central-moment terms will be limited to $r \leqslant 4$ or so.

\section{POLARIZATION PROPERTIES AND EXCITATION MANIFOLDS}

Using the bosonic commutation relation (2), it is possible to rewrite any $r$ th-order product of Stokes operators to a sum of normally ordered terms [34]. Each term has an equal number of creation and annihilation operators, which is not greater than $r$. To exemplify, one can write

$$
\begin{aligned}
\hat{S}_{3}^{2}= & \hat{a}_{H}^{\dagger} \hat{a}_{H}^{\dagger} \hat{a}_{H} \hat{a}_{H}-2 \hat{a}_{H}^{\dagger} \hat{a}_{V}^{\dagger} \hat{a}_{H} \hat{a}_{V}+\hat{a}_{V}^{\dagger} \hat{a}_{V}^{\dagger} \hat{a}_{V} \hat{a}_{V} \\
& +\hat{a}_{H}^{\dagger} \hat{a}_{H}+\hat{a}_{V}^{\dagger} \hat{a}_{V} .
\end{aligned}
$$

As all Stokes operators are composed of terms with one creation and one annihilation operator, this implies that all polarization properties of a state with no excitation above the $N$-photon manifold are determined by the polarization moments up the $r=N$ th order. All moments of order higher than $N$ can have only those normal ordered terms less or equal to the $N$ th order differing from zero, and those terms will always be contained in the moments up to, and including, the $N$ th order. Below we shall see a specific example of this, namely that for a three-photon state, it is sufficient to require that $\left\langle\hat{S}_{\mathbf{n}}^{m}\right\rangle$ is isotropic for $m=1,2,3$ in order for the state to be unpolarized to all orders. Note, however, that should the higher-order central moments be zero, this does not indicate that the state lacks higher-order polarization structure. Instead, the implication is that this structure can be derived from the "beating" terms from lower-order polarization moments, as already hinted in Sec. V.

Another consequence of the fact that a state with no excitation above the $N$-photon manifold has its polarization fully characterized by its $N$ lowest-order moments is that polarization tomography of such a state requires considerably fewer resources than a full-state tomography. For a full-state tomography involving the $(N+1)(N+2) / 2$ basis states (e.g., for $N=1$ the states $|0,0\rangle,|0,1\rangle$, and $|1,0\rangle$ can be chosen) the density matrix is characterized by $N\left(N^{3}+6 N^{2}+13 N+\right.$ 12)/4 independent real numbers. This can be compared to the $N\left(N^{2}+6 N+11\right) / 6$ numbers required in the present method for the polarization tomography of such a state. Raymer et al. have used the term "polarization sector" of the density matrix

for the subset of information needed to characterize only the state's polarization [28].

This said, an $N$-photon state is fully described by $N(N+$ 2) real numbers while the polarization central moments up to, and including, the $r=N$ th order require $N\left(N^{2}+6 N+\right.$ 11)/6 numbers. Very recently we found a method to reduce the number of measurements to the minimum $N(N+2)$, but the analysis and description of this scheme will be published elsewhere [33].

\section{APPLICATION TO DIFFERENT POLARIZATION STATES}

We shall now apply the characterization developed above to a few examples and also compare the theory with experiments in the two-photon excitation manifold. We remind the reader that we use the $\hat{S}_{3}$ eigenstates as our basis states. We shall, first, describe the experimental setup used for the measurements and subsequently describe the polarization properties of some classes of states using the derived methodology. For some states, the theoretical results will be compared to the experimentally obtained data.

\section{A. Polarization measurements and setup}

The experiments were performed by using spatially nondegenerate, photon-pair states generated in the process of spontaneous parametric down-conversion. The photon pairs centered at $780 \mathrm{~nm}$ were generated in a 2-mm-thick type-I $\beta$-barium-borate (BBO) crystal pumped by a femtosecond laser pulse centered at a wavelength of $390 \mathrm{~nm}$. The photon pairs were subsequently filtered by an interference filter with a 4-nm full-width-at-half-maximum (FWHM) bandwidth. The photon pairs were brought to the inputs of a Hong-Ou-Mandel (HOM) interferometer [36], denoted BS in the left part of Fig. 1. When the photons' wave functions overlap in the beam splitter, either the state $|1,1\rangle$ or the state $|2,0\rangle$ can be postselected, depending on the relative polarizations of the incident photons. The two half-wave plates (HWPs) in the state preparation part of the setup are used to prepare the states $|0,2\rangle,|1,1\rangle$, and $|2,0\rangle$. The generation setup is described in more detail in Ref. [37]. In the measurement part of the setup, which is schematically depicted in the right part of Fig. 1, the photon number at each of the two output ports of the polarizing beam splitter (PBS) are measured. The photon

State Preparation

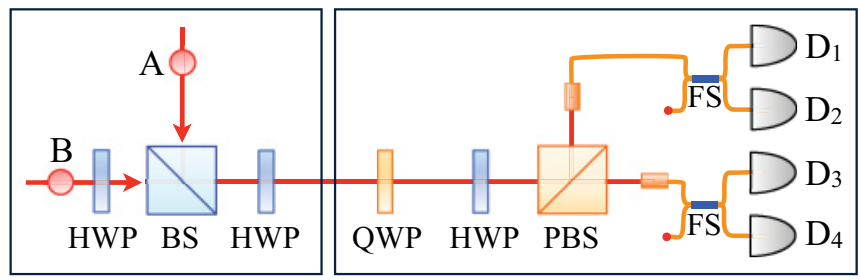

FIG. 1. (Color online) Experimental setup. Single-photons A and $\mathrm{B}$, both horizontally polarized, are prepared by spontaneous parametric down-conversion. (P)BS denotes a (polarization) beam splitter. HWP and QWP denote half-wave and quarter-wave plates, respectively. FS denotes a 50:50 fiber splitter and $D_{1}-D_{4}$ denote single-photon avalanche photo diodes. 

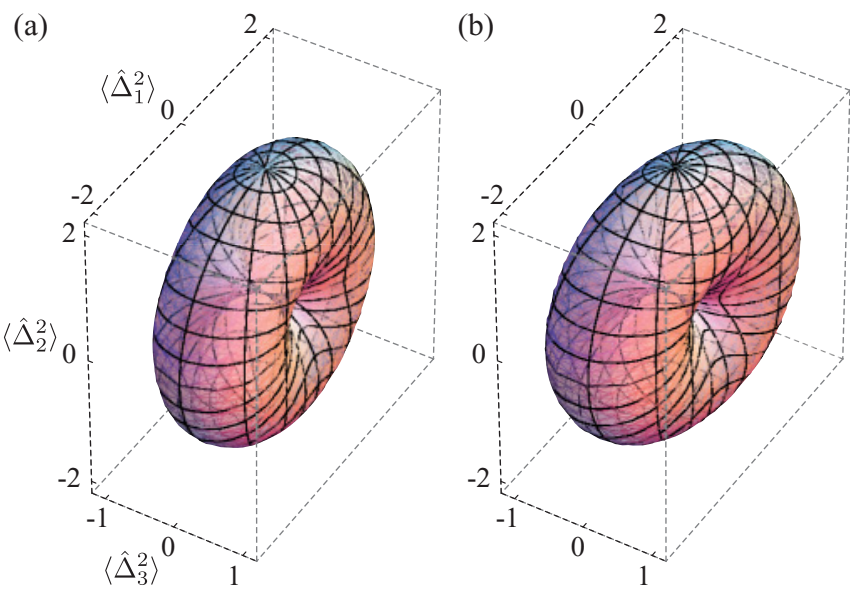

FIG. 2. (Color online) The second-order central moment $\left\langle\hat{\Delta}_{\mathbf{n}}^{2}\right\rangle$ for the state $|2,0\rangle$. Theoretical plot in (a) and the experimental results in (b).

number detectors (resolving the photon numbers 0,1 , and 2 with nonunit efficiency) are realized by concatenating 50:50 fiber beam splitters (FSs) and single-photon detectors $\left(\mathrm{D}_{1}-\mathrm{D}_{4}\right)$. Only in the case when two of the detectors $\mathrm{D}_{1}-\mathrm{D}_{4}$ "click" is the state postselected. Here, the quarter-wave plate (QWP) and HWP before the PBS are used to change the polarization basis of the measurement.

In order to measure the first- and second-order Stokes parameters, a polarizing beam splitter (PBS) is positioned at the measurement stage. At each output of the PBS, a two-photon detector is simulated by a 50:50 fiber beam splitter (FS) and two single-photon detectors (PerkinElmer, SPCMAQRH). The relative coincidence detection efficiencies are estimated from the FS transmittance. Subsequently, the photon detection efficiency of each single-photon detector channel is used to calibrate the measurement of the Stokes parameters. The relative coincidence detection efficiencies of the four detectors are 0.91:0.91:0.82:1 for $|1,1\rangle$ and 0.75:0.76:1:0.57 for $|2,0\rangle$. In order to achieve full information about the first- and second-order Stokes parameters, we measured these coincidences in six distinct measurement bases. For a precise measurement, we measured coincidences three times and each

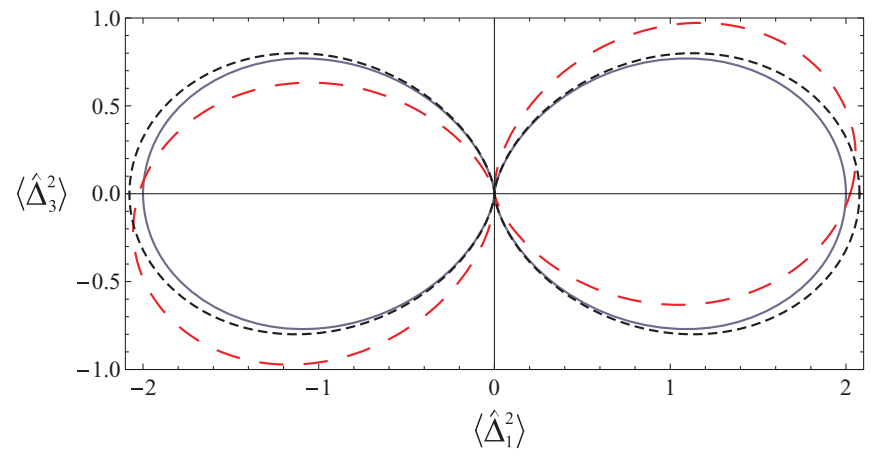

FIG. 3. (Color online) $\left\langle\hat{\Delta}_{\mathbf{n}}^{2}\right\rangle$ in the plane defined by $\left\langle\hat{\Delta}_{2}^{2}\right\rangle=0$ for the state $|2,0\rangle$. The theoretically expected result is a solid (blue) line, the experimentally obtained result is a dashed (red) line, and the experimental result, solidly rotated so its eigenvectors coincide with the intended eigenvectors, is a dotted (black) line.
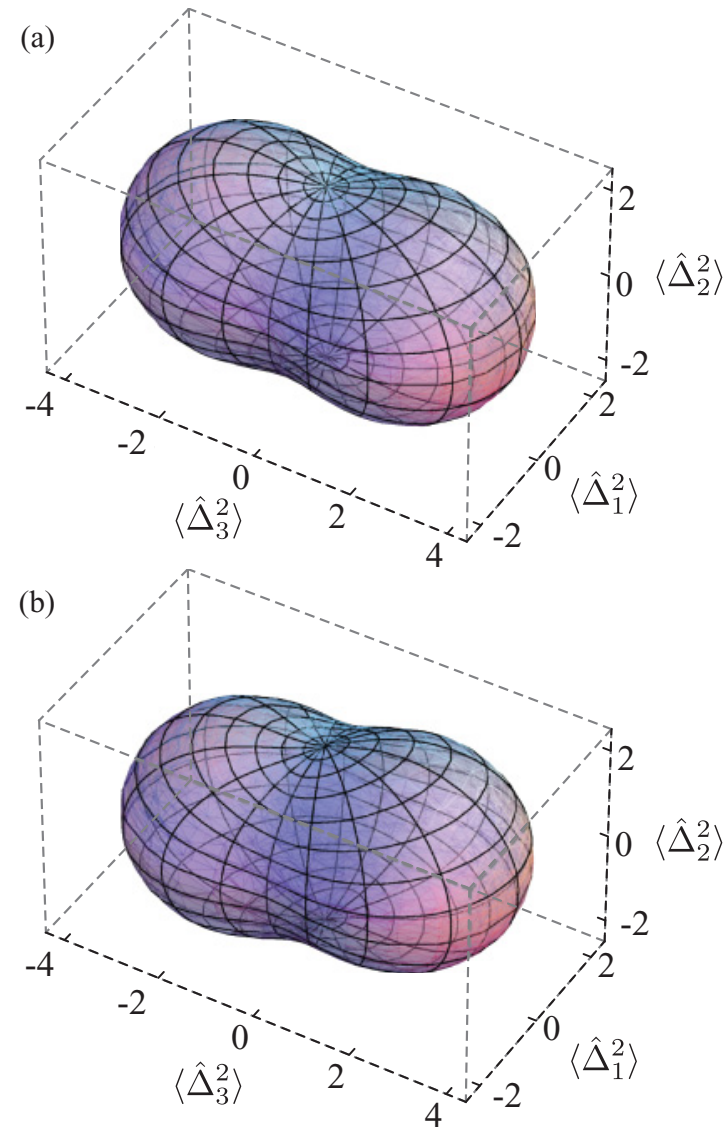

FIG. 4. (Color online) The second-order polarization central moment $\left\langle\hat{\Delta}_{\mathbf{n}}^{2}\right\rangle$ for the state $(|2,0\rangle\langle 2,0|+| 0,2\rangle\langle 0,2|) / 2$. Theoretical plot in (a) and the experimental results in (b).

measurement was done for $3 \mathrm{~s}$. The central moments are obtained from the measured Stokes operator at the six different directions and then solving the equation system generated by Eq. (11). The measured Stokes vector and correlation matrix coefficients Figs. 2-5 are based on are tabulated in the appendix along with the estimated measurement errors. The figures are discussed in Secs. VIII B and VIII F, where the corresponding families of states are treated.

\section{B. SU(2) coherent states}

Through an appropriate polarization transformation of $|N\rangle_{H} \otimes|0\rangle_{V} \equiv|N, 0\rangle$ any $N$-photon, SU(2) coherent state can be obtained. Since a polarization transformation is equivalent to a rotation of the Poincaré sphere, it, thus, suffices to study $|N, 0\rangle$. As all its moments are zero except in excitation manifold $N$ we will suppress this index. The state has $\left\langle\hat{S}_{0}\right\rangle=N$, the Stokes vector is $(0,0, N),\left\langle\hat{\Delta}_{3}^{m}\right\rangle=0$ $\forall m,\left\langle\hat{\Delta}_{1}^{m}\right\rangle=\left\langle\hat{\Delta}_{2}^{m}\right\rangle=0$ for odd $m,\left\langle\hat{\Delta}_{1}^{2}\right\rangle=\left\langle\hat{\Delta}_{2}^{2}\right\rangle=N$, and $\left\langle\hat{\Delta}_{1} \hat{\Delta}_{2}+\hat{\Delta}_{2} \hat{\Delta}_{1}\right\rangle=\left\langle\hat{\Delta}_{1} \hat{\Delta}_{3}+\hat{\Delta}_{3} \hat{\Delta}_{1}\right\rangle=\left\langle\hat{\Delta}_{2} \hat{\Delta}_{3}+\hat{\Delta}_{3} \hat{\Delta}_{2}\right\rangle=0$. Its second-order, polarization central moments are, hence, reduced to a toroidal structure with radius $N$, with its "hole" in the $\hat{S}_{3}$ direction on the Poincaré sphere. Its third-order central moments have the nonvanishing terms $\left\langle\hat{\Delta}_{j}^{2} \hat{\Delta}_{3}+\hat{\Delta}_{j} \hat{\Delta}_{3} \hat{\Delta}_{j}+\hat{\Delta}_{3} \hat{\Delta}_{j}^{2}\right\rangle=-2 N, \quad j \in\{1,2\} . \quad$ The nonvanishing terms of fourth order are $\left\langle\hat{\Delta}_{j}^{4}\right\rangle=3 N^{2}-2 N$, 


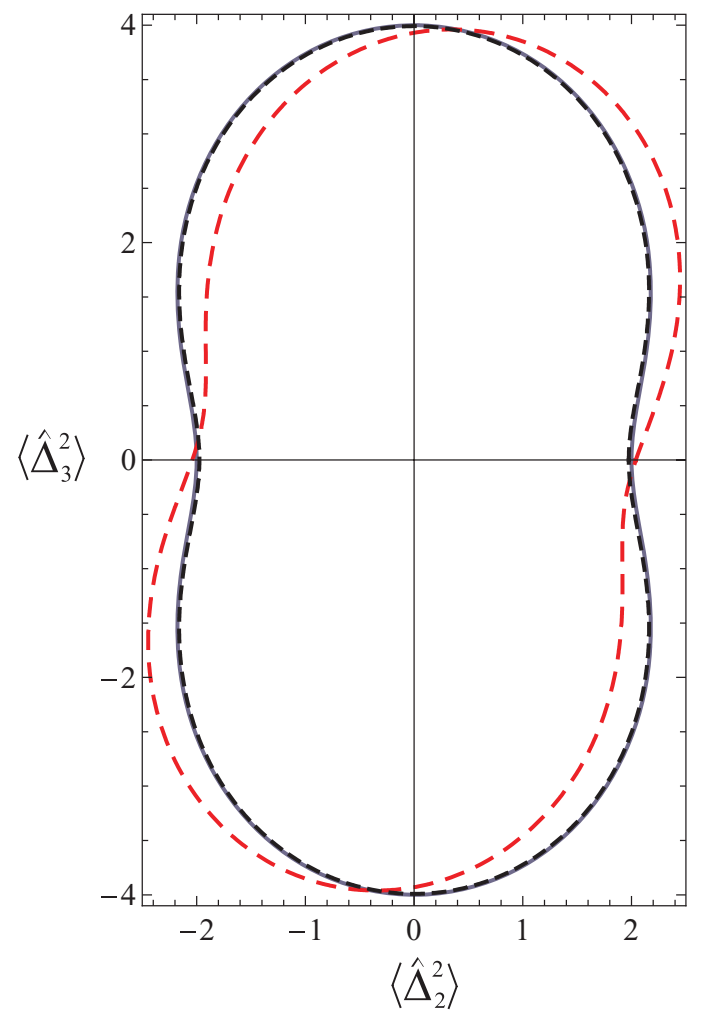

FIG. 5. (Color online) $\left\langle\hat{\Delta}_{n}^{2}\right\rangle$ in the plane defined by $\left\langle\hat{\Delta}_{1}^{2}\right\rangle=0$ for the state $(|2,0\rangle\langle 2,0|+| 0,2\rangle\langle 0,2|) / 2$. The theoretically expected result is a solid (blue) line, the experimentally obtained result is a dashed (red) line, and the experimental result, solidly rotated so its eigenvectors coincide with the intended eigenvectors, is a dotted (black) line.

$\left\langle\hat{\Delta}_{j}^{2} \hat{\Delta}_{3}^{2}+\hat{\Delta}_{3}^{2} \hat{\Delta}_{j}^{2}+\hat{\Delta}_{j} \hat{\Delta}_{3} \hat{\Delta}_{j} \hat{\Delta}_{3}+\hat{\Delta}_{3} \hat{\Delta}_{j} \hat{\Delta}_{3} \hat{\Delta}_{j}+\hat{\Delta}_{j} \hat{\Delta}_{3}^{2} \hat{\Delta}_{j}+\right.$ $\left.\hat{\Delta}_{3} \hat{\Delta}_{j}^{2} \hat{\Delta}_{3}\right\rangle=4 N \quad$ and $\quad\left\langle\hat{\Delta}_{1}^{2} \hat{\Delta}_{2}^{2}+\hat{\Delta}_{2}^{2} \hat{\Delta}_{1}^{2}+\hat{\Delta}_{1} \hat{\Delta}_{2} \hat{\Delta}_{1} \hat{\Delta}_{2}+\right.$ $\left.\hat{\Delta}_{2} \hat{\Delta}_{1} \hat{\Delta}_{2} \hat{\Delta}_{1}+\hat{\Delta}_{1} \hat{\Delta}_{2}^{2} \hat{\Delta}_{1}+\hat{\Delta}_{2} \hat{\Delta}_{1}^{2} \hat{\Delta}_{2}\right\rangle=6 N^{2}-4 N, j \in\{1,2\}$. This is a minimum-sum uncertainty-state [saturating the left inequality in Eq. (4)]. In Fig. 2 we plot the theoretically computed function $\left\langle\hat{\Delta}_{\mathbf{n}}^{2}\right\rangle_{2}$ for the state $|2,0\rangle$ to the left, and the experimentally obtained results on the right. The measured Stokes vector of this state is $(-0.19,0.12,1.97)$. The main source of error in the estimation of the Stokes vector is neither fluctuations nor random errors, but the fact that the generated state and the measurement axes are slightly rotated relative to each other. This is better seen in Fig. 3, where we have plotted the theoretically expected result (solid) and the experimental figure (dashed) as derived directly from the measured parameters in Table I (no fitting done) in the $\hat{S}_{1}-\hat{S}_{3}$ plane. We have used the measurement data and computed the eigenvectors and eigenvalues for the matrix $\boldsymbol{\Gamma}_{2}$ defined in Eq. (13). The eigenvalues (that, due to the Hermiticity of $\boldsymbol{\Gamma}_{2}$, are the plot's extrema, which are found at orthogonal points on the Poincaré sphere) are 2.08, 2.02, and 0.00, respectively, and, e.g., the eigenvector corresponding to the smallest eigenvalue is $(-0.15,-0.02,0.99)$. This eigenvector is rotated about $8.1^{\circ}$ from the $\hat{S}_{3}$ axis, mainly around the $\hat{S}_{2}$ axis (roughly in agreement with the orientation of the state's Stokes vector). If we replot the figure, with no other "fitting" than a solid rotation of the experimental figure to make its eigenvectors coincide with the Poincaré sphere coordinate axes, we obtain the dotted curve in Fig. 3. Note that the needed rotation is not exactly perpendicular to the drawn plane, so the rotated figure will also change shape slightly. The dotted figure (and the $\boldsymbol{\Gamma}_{2}$ eigenvalues) confirms that the experimental errors due to fluctuations are below $\pm 4 \%$. The main errors are systematic, due to imperfect polarization optics and beam splitters. The systematic errors will persist even if additional points on the Poincaré sphere are measured, indicating that the proposed method is "efficient" from a data-collecting point of view. By measuring the nine data in Table I one can, hence, estimate both the accuracy and the precision of the experiment.

\section{C. $|N, N\rangle$ states}

This state has $\left\langle\hat{S}_{0}\right\rangle=2 N$, the Stokes vector is $(0,0,0)$, and $\left\langle\hat{\Delta}_{3}^{m}\right\rangle=0 \forall m$. The only nonvanishing second-order, central-moment terms are $\left\langle\hat{\Delta}_{j}^{2}\right\rangle=2 N(N+1)$, $j \in\{1,2\}$. The state has vanishing third-order central moment in every direction, and its fourth-order, nonvanishing central-moment terms are $\left\langle\hat{\Delta}_{j}^{4}\right\rangle=$ $2 N\left(3 N^{3}+6 N^{2}+N-2\right),\left\langle\hat{\Delta}_{j}^{2} \hat{\Delta}_{3}^{2}+\hat{\Delta}_{3}^{2} \hat{\Delta}_{j}^{2}+\hat{\Delta}_{j} \hat{\Delta}_{3} \hat{\Delta}_{j} \hat{\Delta}_{3}+\right.$ $\left.\hat{\Delta}_{3} \hat{\Delta}_{j} \hat{\Delta}_{3} \hat{\Delta}_{j}+\hat{\Delta}_{j} \hat{\Delta}_{3}^{2} \hat{\Delta}_{j}+\hat{\Delta}_{3} \hat{\Delta}_{j}^{2} \hat{\Delta}_{3}\right\rangle=8 N(N+1), \quad$ and $\left\langle\hat{\Delta}_{1}^{2} \hat{\Delta}_{2}^{2}+\hat{\Delta}_{2}^{2} \hat{\Delta}_{1}^{2}+\hat{\Delta}_{1} \hat{\Delta}_{2} \hat{\Delta}_{1} \hat{\Delta}_{2}+\hat{\Delta}_{2} \hat{\Delta}_{1} \hat{\Delta}_{2} \hat{\Delta}_{1}+\hat{\Delta}_{1} \hat{\Delta}_{2}^{2} \hat{\Delta}_{1}+\right.$ $\left.\hat{\Delta}_{2} \hat{\Delta}_{1}^{2} \hat{\Delta}_{2}\right\rangle=4 N\left(3 N^{3}+6 N^{2}+N-2\right), j \in\{1,2\}$. This is a pure, maximum-uncertainty state, saturating the right inequality in Eq. (4). If one plots the experimentally obtained results one obtains a figure very similar to Fig. 2(b), except that $\left\langle\hat{\Delta}_{\mathbf{n}}^{2}\right\rangle_{2}$ for this state is approximately twice as large in all directions as for the state $|2,0\rangle$. The measured Stokes vector of this state is $(-0.01,-0.08,0.01)$. Extracting the measured extremal values for $\left\langle\hat{\Delta}_{\mathbf{n}}^{2}\right\rangle_{2}$ from the experimental

TABLE I. The experimental data for the state $|2,0\rangle$.

\begin{tabular}{|c|c|c|c|c|}
\hline \multirow[b]{2}{*}{ Operator } & \multicolumn{2}{|c|}{ Theory } & \multicolumn{2}{|c|}{ Experiment } \\
\hline & First order & Second order & First order & Second order \\
\hline$\left\langle\hat{S}_{1}\right\rangle$ & 0 & 2 & $-0.19 \pm 0.06$ & $2.06 \pm 0.03$ \\
\hline$\left\langle\hat{S}_{2}\right\rangle$ & 0 & 2 & $0.12 \pm 0.04$ & $2.04 \pm 0.02$ \\
\hline$\left\langle\hat{S}_{3}\right\rangle$ & 2 & 4 & $1.97 \pm 0.01$ & $3.93 \pm 0.02$ \\
\hline$\left\langle\hat{S}_{1} \hat{S}_{2}+\hat{S}_{2} \hat{S}_{1}\right\rangle$ & - & 0 & - & $-0.09 \pm 0.10$ \\
\hline$\left\langle\hat{S}_{2} \hat{S}_{3}+\hat{S}_{3} \hat{S}_{2}\right\rangle$ & - & 0 & - & $0.54 \pm 0.08$ \\
\hline$\left\langle\hat{S}_{3} \hat{S}_{1}+\hat{S}_{1} \hat{S}_{3}\right\rangle$ & - & 0 & - & $-0.13 \pm 0.15$ \\
\hline
\end{tabular}


TABLE II. The experimental data for the state $(|2,0\rangle\langle 2,0|+| 0,2\rangle\langle 0,2|) / 2$.

\begin{tabular}{lccrrr}
\hline \hline & \multicolumn{2}{c}{ Theory } & & \multicolumn{2}{c}{ Experiment } \\
\cline { 2 - 3 } Operator & First order & Second order & & First order & Second order \\
\hline$\left\langle\hat{S}_{1}\right\rangle$ & 0 & 2 & & $-0.11 \pm 0.04$ & $2.04 \pm 0.04$ \\
$\left\langle\hat{S}_{2}\right\rangle$ & 0 & 2 & & $-0.10 \pm 0.03$ & $2.05 \pm 0.04$ \\
$\left\langle\hat{S}_{3}\right\rangle$ & 0 & 0 & & $0.00 \pm 0.01$ & $3.93 \pm 0.02$ \\
$\left\langle\hat{S}_{1} \hat{S}_{2}+\hat{S}_{2} \hat{S}_{1}\right\rangle$ & - & 0 & - & $-0.01 \pm 0.11$ \\
$\left\langle\hat{S}_{2} \hat{S}_{3}+\hat{S}_{3} \hat{S}_{2}\right\rangle$ & - & 0 & - & $-0.69 \pm 0.07$ \\
$\left\langle\hat{S}_{3} \hat{S}_{1}+\hat{S}_{1} \hat{S}_{3}\right\rangle$ & - & $0.01 \pm 0.11$ \\
\hline \hline
\end{tabular}

data (i.e., the eigenvalues of $\boldsymbol{\Gamma}_{2}$ ), one obtains 4.10, 3.98, and -0.03 , indicating an experimental error due to fluctuations below $\pm 3 \%$. This data too, indicates a slight mismatch (about $12^{\circ}$ ) between the state's intended orientation on the Poincaré sphere and its measured orientation. Note that the state's orientation cannot be obtained from the Stokes vector, since nominally it vanishes. The measured values are simply the measurement errors. However, from the second-order moment data the state's orientation relative to the Poincaré sphere axes can be well determined.

\section{Two-mode coherent states}

Any two-mode, coherent state $\left|\alpha^{\prime}, \alpha^{\prime \prime}\right\rangle$ can be converted into the state ||$\alpha|, 0\rangle$, where $|\alpha|^{2}=\left|\alpha^{\prime}\right|^{2}+\left|\alpha^{\prime \prime}\right|^{2}$, by a polarization transformation. Therefore, it suffices to study the latter state, which can be written

$$
\exp \left(-|\alpha|^{2} / 2\right) \sum_{N=0}^{\infty} \frac{|\alpha|^{N}}{\sqrt{N !}}|N, 0\rangle .
$$

In each excitation manifold (except the nonexcited one), the state has the same central moments as an SU(2) coherent state. Summing over the manifolds, the coherent state has $\left\langle\hat{S}_{0}\right\rangle=$ $|\alpha|^{2}$, the Stokes vector $\left(0,0,|\alpha|^{2}\right)$ and $\left\langle\hat{\Delta}_{j}^{2}\right\rangle=|\alpha|^{2}$ for $j \in$ $\{1,2,3\}$. The off-diagonal coefficients of the covariance matrix $\boldsymbol{\Gamma}$ are zero, so the second-order central moment is isotropic with radius $|\alpha|^{2}$. In the third order, the only nonvanishing central-moment terms are $\left\langle\hat{\Delta}_{3}^{3}\right\rangle=\left\langle\hat{\Delta}_{1}^{2} \hat{\Delta}_{3}+\hat{\Delta}_{1} \hat{\Delta}_{3} \hat{\Delta}_{1}+\right.$ $\left.\hat{\Delta}_{3} \hat{\Delta}_{1}^{2}\right\rangle=\left\langle\hat{\Delta}_{2}^{2} \hat{\Delta}_{3}+\hat{\Delta}_{2} \hat{\Delta}_{3} \hat{\Delta}_{2}+\hat{\Delta}_{3} \hat{\Delta}_{2}^{2}\right\rangle=|\alpha|^{2}$. The nonvanishing fourth-order central-moment terms are

$$
\left\langle\hat{\Delta}_{j}^{4}\right\rangle=3|\alpha|^{4}+|\alpha|^{2}
$$

for $j \in\{1,2,3\}$ and

$$
\begin{gathered}
\left\langle\hat{\Delta}_{j}^{2} \hat{\Delta}_{k}^{2}+\hat{\Delta}_{j} \hat{\Delta}_{k} \hat{\Delta}_{j} \hat{\Delta}_{k}+\hat{\Delta}_{j} \hat{\Delta}_{k}^{2} \hat{\Delta}_{j}+\hat{\Delta}_{k} \hat{\Delta}_{j}^{2} \hat{\Delta}_{k}\right. \\
\left.+\hat{\Delta}_{k} \hat{\Delta}_{j} \hat{\Delta}_{k} \hat{\Delta}_{j}+\hat{\Delta}_{k}^{2} \hat{\Delta}_{j}^{2}\right\rangle=6|\alpha|^{4}+2|\alpha|^{2}
\end{gathered}
$$

for $j, k \in\{1,2,3\}$ and $j<k$.

\section{E. Unpolarized states, SU(2)-invariant states, and thermal states}

The unpolarized states [30,33] have isotropic central moments for all orders. Due to symmetry, the odd-order central moments are identically zero. For an $N$-photon unpolarized state, the second-order central moment in any direction is $\left\langle\hat{\Delta}_{\mathbf{n}}^{2}\right\rangle=\left\langle\hat{S}_{\mathbf{n}}^{2}\right\rangle=N(N+2) / 3$, and the fourth-order central moment is $\left\langle\hat{\Delta}_{\mathbf{n}}^{4}\right\rangle=N(N+2)\left(3 N^{2}+6 N-4\right) / 15$.
SU(2)-invariant states [38] constitute a subclass of the unpolarized states [30]. They take the form

$$
\sum_{N=0}^{\infty} \frac{p_{N}}{N+1} \hat{\mathbb{1}}_{N}
$$

where $p_{N}$ is a probability distribution and $\hat{\mathbb{1}}_{N}$ is the identity operator in the $N$ th manifold. A simple example of a state that is unpolarized but not $\mathrm{SU}(2)$ invariant is given in Ref. [33]. In Table II we have tabulated the measured data for the unpolarized [and SU(2)-invariant] two-photon state $(|2,0\rangle\langle 2,0|+$ $|1,1\rangle\langle 1,1|+| 0,2\rangle\langle 0,2|) / 3$. The measured Stokes vector for this state is $(-0.07,-0.10,0.01)$. As can be read essentially directly from the table, the experimentally obtained function $\left\langle\hat{\Delta}_{\mathbf{n}}^{2}\right\rangle$ is more or less a sphere. The experimentally obtained extremal values of the function are 2.72, 2.68, and 2.62, indicating an error due to fluctuations of about $\pm 2 \%$.

The thermal states, finally, constitute a subclass of the $\mathrm{SU}(2)$-invariant states with $p_{N}=\bar{N}^{N} /(1+\bar{N})^{N+1}$, where $\bar{N}$ is the average excitation. That is, $\bar{N}=[\exp (h v / k T)-1]^{-1}$, where $h$ is Planck's constant, $v$ is the optical frequency, $k$ Boltzmann's constant, and $T$ the temperature. Their secondand fourth-order central moments are given above, and their excitation probability averaged second- and fourth-order central moments are $\bar{N}+2 \bar{N}^{2} / 3$ and $\bar{N}+26 \bar{N}^{2} / 3+12 \bar{N}^{3}+$ $24 \bar{N}^{4} / 5$, respectively.

\section{F. A two-photon mixed state}

In our last experiment, we generated and analyzed the $(|2,0\rangle\langle 2,0|+| 0,2\rangle\langle 0,2|) / 2$. Its second-order central moment $\left\langle\hat{\Delta}_{\mathbf{n}}^{2}\right\rangle_{2}$ is depicted in Fig. 4 (theory on the left, experiments on the right). The measured Stokes vector of this state is $(-0.11,-0.10,0.00)$.

To better appreciate the random and the systematic errors of the measurement, a cut through Fig. 4 in the $\left\langle\hat{\Delta}_{1}^{2}\right\rangle=0$ plane is shown in Fig. 5. Again, it is seen that the experimentally obtained figure is not aligned with the intended orientation. Instead, it is rotated about $10^{\circ}$ around the $\hat{S}_{1}$ axis. The figure's extremal values are 3.99, 2.03, and 1.97. Reorienting the figure by a solid rotation so the measured and intended axes coincide, one obtains the dotted curve in Fig. 5. By removing the systematic errors in such a way, one obtains a very good $( \pm 2 \%)$ agreement between the experiments and the theory. 


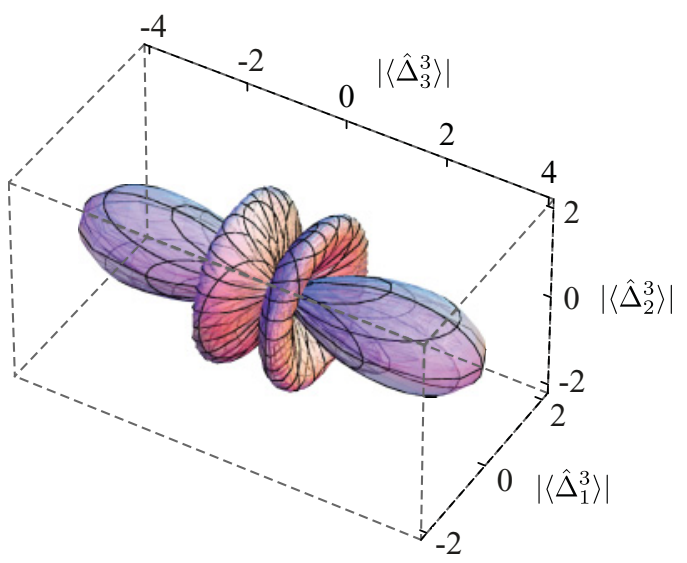

FIG. 6. (Color online) The absolute value of $\left\langle\hat{\Delta}_{\mathbf{n}}^{3}\right\rangle_{3}$ for the state $\frac{1}{3}|3,0\rangle\left\langle 3,0\left|+\frac{1}{2}\right| 1,2\right\rangle\left\langle 1,2\left|+\frac{1}{6}\right| 0,3\right\rangle\langle 0,3|$.

\section{G. Some three-photon states}

By mixing three-photon states, one can get quite complicated polarization characteristics. Here we explore the polarization characteristics of a few states up to the third order and show that for $N=3$ there exist six classes of states if they are sorted according to their first-, second-, and third-order polarization central moments. Before giving examples of the classes, it is helpful to retain the uncertainty relation (4). In the third excitation manifold this means that the sum of the second-order polarization variances must lie between the values 6 and 15. In order to have an isotropic second-order central moment, the diagonalized covariance

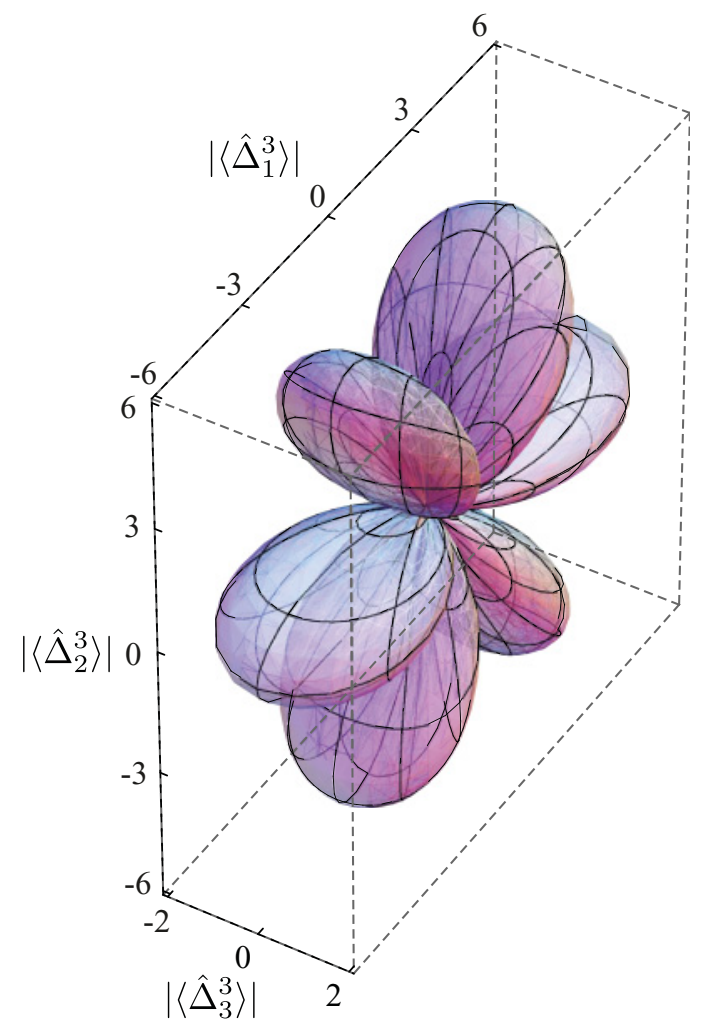

FIG. 7. (Color online) The absolute value of $\left\langle\hat{\Delta}_{\mathbf{n}}^{3}\right\rangle_{3}$ for the state $(|0,3\rangle+|3,0\rangle) / \sqrt{2}$. matrix $\Gamma$ should be proportional to the $3 \times 3$ unit matrix, and the relation above dictates that the proportionality factor must be in the range between 2 to 5 . In fact, only minimum sum uncertainty states will reach the lower limit in Eq. (4) and such states have an anisotropic second-order polarization central moment. We conjecture that the lower limit for an isotropic second-order central moment is in fact $\left\langle\hat{S}_{0}\right\rangle$ so the minimum uncertainty sum for second-order isotropic states is $3\left\langle\hat{S}_{0}\right\rangle$ (and, specifically, 9 for three-photon states). The corresponding state is $|N, 0\rangle\langle N, 0|\left(1 \pm[\{N-1\} / N]^{1 / 2}\right) / 2+$ $|0, N\rangle\langle 0, N|\left(1 \mp[\{N-1\} / N]^{1 / 2}\right) / 2$, and in this specific manifold $\left(1 / 2 \pm 6^{-1 / 2}\right)|3,0\rangle\left\langle 3,0\left|+\left(1 / 2 \mp 6^{-1 / 2}\right)\right| 0,3\right\rangle\langle 0,3|$.

Since states that lack first-order polarization but are secondorder polarized have already been discussed, we shall now look at states that have an isotropic, second-order polarization central moment but that may have higher-order polarization structure. Applying the requirements for a state to have isotropic polarization up to second order, one can derive such a three-photon state's density matrix $\hat{\varrho}$ to be of the form

$$
\hat{\rho}=\left(\begin{array}{cccc}
\rho_{11} & \rho_{12} & \rho_{13} & \rho_{14} \\
\rho_{12}^{*} & 1-3 \rho_{11} & -\sqrt{3} \rho_{12} & -\rho_{13} \\
\rho_{13}^{*} & -\sqrt{3} \rho_{12}^{*} & 3 \rho_{11}-\frac{1}{2} & \rho_{12} \\
\rho_{14}^{*} & -\rho_{13}^{*} & \rho_{12}^{*} & \frac{1}{2}-\rho_{11}
\end{array}\right) .
$$

Here, $\rho_{11}$ is real and subject to the restriction $1 / 6 \leqslant \rho_{11} \leqslant 1 / 3$, whereas $\rho_{12}, \rho_{13}$, and $\rho_{14}$ may be complex. Of course, the general coherence property for the off-diagonal coefficients $\left|\rho_{j k}\right| \leqslant \sqrt{\rho_{j j} \rho_{k k}}$ holds and imposes additional (but simple) restrictions on the matrix, once one has chosen $\rho_{11}$.

Note that the matrix above defines the sufficient conditions for a density matrix to have vanishing first- and second-order central moments, but that it does not include all necessary conditions to make it a density matrix. That is, it may be that, for certain choices of parameters, the matrix is not strictly non-negative. Hence, the reader is warned when using Eq. (22), to make sure that the ensuing matrix is non-negative.

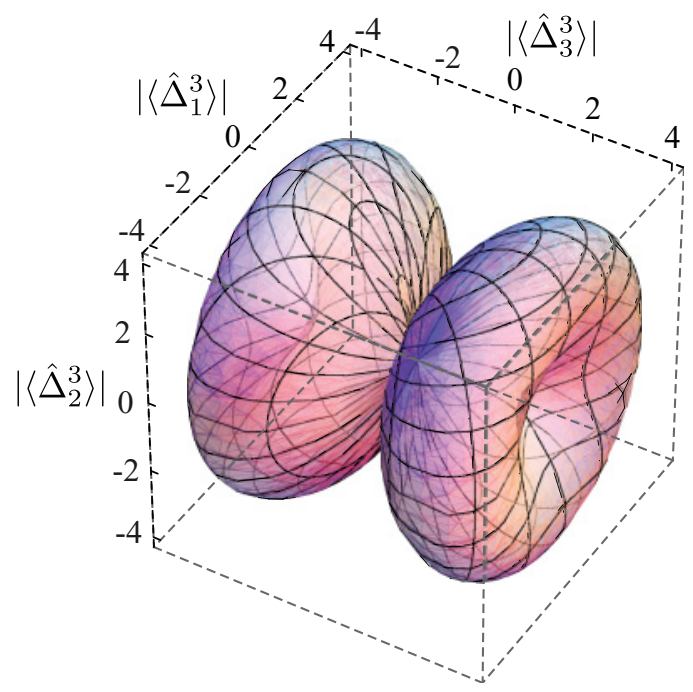

FIG. 8. (Color online) The absolute value of $\left\langle\hat{\Delta}_{\mathbf{n}}^{3}\right\rangle_{3}$ for the state $\frac{19}{36}|3,0\rangle\left\langle 3,0\left|+\frac{15}{36}\right| 1,2\right\rangle\left\langle 1,2\left|+\frac{1}{18}\right| 0,3\right\rangle\langle 0,3|$. 
TABLE III. States exemplifying the six different three-photon polarization classes.

\begin{tabular}{llcr}
\hline \hline State & \multicolumn{3}{c}{ Polarization } \\
\cline { 2 - 3 } & $\left\langle\hat{S}_{\mathbf{n}}\right\rangle_{3}$ & $\left\langle\hat{\Delta}_{\mathbf{n}}^{2}\right\rangle_{3}$ & $\left\langle\hat{\Delta}_{\mathbf{n}}^{3}\right\rangle_{3}$ \\
\hline$\hat{\mathbb{1}} / 4$ & Yes & Yes & Yes \\
$\frac{1}{3}|3,0\rangle\left\langle 3,0\left|+\frac{1}{2}\right| 1,2\right\rangle\left\langle 1,2\left|+\frac{1}{6}\right| 0,3\right\rangle\langle 0,3|$ & Yes & Yes & No \\
$\frac{1}{2}(|3,0\rangle\langle 3,0|+| 0,3\rangle\langle 0,3|)$ & Yes & No & Yes \\
$(|3,0\rangle+|0,3\rangle) / \sqrt{2}$ & Yes & No & No \\
$\frac{19}{36}|3,0\rangle\left\langle 3,0\left|+\frac{15}{36}\right| 1,2\right\rangle\left\langle 1,2\left|+\frac{1}{18}\right| 0,3\right\rangle\langle 0,3|$ & No & Yes & No \\
$|3,0\rangle$ & No & No & No \\
\hline \hline
\end{tabular}

For $\hat{\varrho}$ of the form in Eq. (22) one finds that $\langle\hat{\mathbf{S}}\rangle_{3}=(0,0,0)$, that $\left\langle\hat{\Delta}_{i}^{2}\right\rangle_{3}=5$ for $i=1,2,3$, and, hence, that

$$
\Gamma_{3}=\left(\begin{array}{lll}
5 & 0 & 0 \\
0 & 5 & 0 \\
0 & 0 & 5
\end{array}\right) .
$$

One can also deduce from (22) that there is no pure, threephoton state that is unpolarized to second order. This follows from the condition that, for a pure state, $\left|\varrho_{j k}\right|^{2}=\varrho_{j j} \varrho_{k k}$. Applied to the density matrix (22), one gets the three conditions $\left|\varrho_{12}\right|^{2}=\varrho_{11}\left(1-3 \varrho_{11}\right), 3\left|\varrho_{12}\right|^{2}=\left(1-3 \varrho_{11}\right)\left(3 \varrho_{11}-\frac{1}{2}\right)$, and $\left|\varrho_{12}\right|^{2}=\left(3 \varrho_{11}-\frac{1}{2}\right)\left(\frac{1}{2}-\varrho_{11}\right)$. The first two of these equations demand that $\varrho_{11}=1 / 3 \Rightarrow \varrho_{12}=0$, but this value does not satisfy the third equation.

An already discussed class of states are the unpolarized states. This is the smallest class of three-photon states, because there is only one such three-photon state. The state has, of course, isotropic polarization properties of all orders, but requiring this property for only the lowest three orders uniquely singles out this state.

The mixed state $\frac{1}{3}|3,0\rangle\left\langle 3,0\left|+\frac{1}{2}\right| 1,2\right\rangle\left\langle 1,2\left|+\frac{1}{6}\right| 0,3\right\rangle\langle 0,3|$ lacks first-order polarization, has $\left\langle\hat{\Delta}_{\mathbf{n}}^{2}\right\rangle_{3}=5$, but has thirdorder polarization structure. Its third-order polarization central moment is shown in Fig. 6. This state is, thus, unpolarized to second order.

The mixed state $(|3,0\rangle\langle 3,0|+| 0,3\rangle\langle 0,3|) / 2$ has vanishing first- and third-order central moments in all directions but has an anisotropic second-order polarization central moment, with the predominant fluctuations along the $\hat{S}_{3}$ axis. $\left\langle\hat{\Delta}_{\mathbf{n}}^{2}\right\rangle_{3}$ has a "peanut" shape (similar to Fig. 4) with "semiaxes" lengths $\left\langle\hat{\Delta}_{1}^{2}\right\rangle_{3}=\left\langle\hat{\Delta}_{2}^{2}\right\rangle_{3}=3$ and $\left\langle\hat{\Delta}_{3}^{2}\right\rangle_{3}=9$. This is, thus, a maximum uncertainty state.
The pure state $(|0,3\rangle+|3,0\rangle) / \sqrt{2}$ lacks first-order polarization, has $\left\langle\hat{\Delta}_{\mathbf{n}}^{2}\right\rangle_{3}$ identical to the $(|3,0\rangle\langle 3,0|+| 0,3\rangle\langle 0,3|) / 2$ mixed state, and its third-order polarization central moment is shown in Fig. 7. It is also a maximum uncertainty state.

Changing the mixing ratios somewhat, one finds that the mixed state $\frac{7}{18}|3,0\rangle\left\langle 3,0\left|+\frac{1}{3}\right| 1,2\right\rangle\left\langle 1,2\left|+\frac{5}{18}\right| 0,3\right\rangle\langle 0,3|$ also has no first-order polarization but second- and third-order structure. The second-order central moment is again "peanut shaped" with "half-axes lengths" $(19,13,13) / 3$, and $\left\langle\hat{\Delta}_{\mathbf{n}}^{3}\right\rangle_{3}$ is similar to that in Fig. 6. Hence, comparing this state with the state $(|0,3\rangle+|3,0\rangle) / \sqrt{2}$ they have very similar polarization properties in the first two orders (and both are maximum uncertainty states), but their third-order properties differ vastly.

Finally, an example of a state that has first- and third-order polarization structure but has an isotropic second-order central moment is the mixed state $\frac{19}{36}|3,0\rangle\left\langle 3,0\left|+\frac{15}{36}\right| 1,2\right\rangle\langle 1,2|+$ $\frac{1}{18}|0,3\rangle\langle 0,3|$. This state has the Stokes vector $(-1,0,0)$, $\left\langle\hat{\Delta}_{\mathbf{n}}^{2}\right\rangle_{3}=14 / 3$, and $\left\langle\hat{\Delta}_{\mathbf{n}}^{3}\right\rangle_{3}$ is shown in Fig. 8 .

It is not possible to find three-photon states that have first-order polarization but a vanishing third-order polarization central moment. The above list of possible polarization classes exhausts all the combinations of polarization structures up to third order and shows that six different classes exist out of the total of eight a priori possible combinations. The classes, with example of associated states, are tabulated in Table III.

\section{CONCLUSIONS}

We have developed a systematic method, using central moments, for assessing the polarization characteristics of quantized fields. The method goes well beyond the "standard" one that only considers first-order moments and that, moreover, averages over the excitation manifolds. We have shown that

TABLE IV. The experimental data for the state $|1,1\rangle$.

\begin{tabular}{lcccrr}
\hline \hline & \multicolumn{2}{c}{ Theory } & & \multicolumn{2}{c}{ Experiment } \\
\cline { 2 - 3 } Operator & First order & Second order & & First order & Second order \\
\hline$\left\langle\hat{S}_{1}\right\rangle$ & 0 & 4 & & $-0.01 \pm 0.04$ & $3.98 \pm 0.00$ \\
$\left\langle\hat{S}_{2}\right\rangle$ & 0 & 4 & & $-0.08 \pm 0.03$ & $3.93 \pm 0.03$ \\
$\left\langle\hat{S}_{3}\right\rangle$ & 0 & 0 & & $0.01 \pm 0.02$ & $0.15 \pm 0.05$ \\
$\left\langle\hat{S}_{1} \hat{S}_{2}+\hat{S}_{2} \hat{S}_{1}\right\rangle$ & - & 0 & - & $0.03 \pm 0.02$ \\
$\left\langle\hat{S}_{2} \hat{S}_{3}+\hat{S}_{3} \hat{S}_{2}\right\rangle$ & - & 0 & & - & $-1.67 \pm 0.14$ \\
$\left\langle\hat{S}_{3} \hat{S}_{1}+\hat{S}_{1} \hat{S}_{3}\right\rangle$ & - & 0 & & - & $0.12 \pm 0.16$ \\
\hline \hline
\end{tabular}


TABLE V. The experimental data for the state $(|2,0\rangle\langle 2,0|+| 1,1\rangle\langle 1,1|+| 0,2\rangle\langle 0,2|) / 3$.

\begin{tabular}{lcccrr}
\hline \hline & \multicolumn{2}{c}{ Theory } & & \multicolumn{2}{c}{ Experiment } \\
\cline { 2 - 3 } Operator & First order & Second order & & First order & Second order \\
\hline$\left\langle\hat{S}_{1}\right\rangle$ & 0 & $8 / 3$ & & $-0.07 \pm 0.03$ & $2.69 \pm 0.03$ \\
$\left\langle\hat{S}_{2}\right\rangle$ & 0 & $8 / 3$ & & $-0.10 \pm 0.02$ & $2.68 \pm 0.03$ \\
$\left\langle\hat{S}_{3}\right\rangle$ & 0 & $8 / 3$ & & $0.01 \pm 0.01$ & $2.67 \pm 0.02$ \\
$\left\langle\hat{S}_{1} \hat{S}_{2}+\hat{S}_{2} \hat{S}_{1}\right\rangle$ & - & 0 & - & $0.00 \pm 0.07$ \\
$\left\langle\hat{S}_{2} \hat{S}_{3}+\hat{S}_{3} \hat{S}_{2}\right\rangle$ & - & 0 & & - & $-0.09 \pm 0.06$ \\
$\left\langle\hat{S}_{3} \hat{S}_{1}+\hat{S}_{1} \hat{S}_{3}\right\rangle$ & - & 0 & & - & $0.04 \pm 0.09$ \\
\hline \hline
\end{tabular}

there exist a rich 'zoo' of polarization states, including, e.g., states that are unpolarized up to a given order but that have higher-order structure (so-called hidden polarization). However, as expected, for most states the polarization characteristics are dominated by the first- and second-order behavior, as higher-order polarization moments always contain "beating terms" originating from lower orders. Some states, however, show polarization structure that is dominated by higher-order moments, and examples of such states are given.

The suggested method is not the only way to fully characterize the polarization of quantized fields. In particular, two more-or-less equivalent methods are mentioned, namely generalized coherence matrices $[19,34]$ and the expectation values of all combinations of Stokes operators [33].

Since a state is not fully specified by its polarization properties, it comes as no surprise that polarization tomography is less resource demanding than full-state tomography. We have quantified this difference and indicated a "recipe" for determining polarization properties up to a certain order.

\section{ACKNOWLEDGMENTS}

We thank G. Leuchs for useful discussions. Financial support from the Swedish Foundation for International
Cooperation in Research and Higher Education (STINT), the Swedish Research Council (VR) through its Linnæus Center of Excellence ADOPT and Contract No. 319-2010-7332, the National Research Foundation of Korea (2009-0070668 and 2011-0021452), Spanish DGI (Grants No. FIS2008-04356 and No. FIS2011-26786), the UCM-BSCH program (Grant No. GR-920992), and the CONACyT (Grant No. 106525).

\section{APPENDIX}

In Tables I-II and IV-V, the measurement data for some states and the estimated errors due to fluctuations are presented along with the theoretical predictions. As can be seen, what looks like measurement errors far exceeding the estimated "error bars" (e.g., for $\left\langle\hat{S}_{2} \hat{S}_{3}+\hat{S}_{3} \hat{S}_{2}\right\rangle$ of state $|2,0\rangle$ ) are actually systematic errors due to imperfect polarization optics. These can be corrected by properly aligning the experimental Poincaré axes with the "theoretical" axes. When this is done, as is shown in Figs. 3 and 5, the experimental figures are coinciding to within a few percentages with the theoretical ones, showing that the errors due to fluctuations are relatively modest and within the relative range indicated by the estimated error limits in the tables.
[1] G. G. Stokes, Trans. Cambridge Philos. Soc. 9, 399 (1852).

[2] T. Carozzi, R. Karlsson, and J. Bergman, Phys. Rev. E 61, 2024 (2000).

[3] T. Setälä, K. Lindfors, M. Kaivola, J. Tervo, and A. T. Friberg, Opt. Lett. 29, 2587 (2004).

[4] A. Luis, Phys. Rev. A 71, 063815 (2005).

[5] J. J. Gil, Eur. Phys. J. Appl. Phys. 40, 1 (2007).

[6] V. P. Karassiov, J. Phys. A 26, 4345 (1993).

[7] A. V. Burlakov and D. N. Klyshko, JETP Lett. 69, 839 (1999).

[8] T. Sh. Iskhakov, M. V. Chekhova, G. O. Rytikov, and G. Leuchs, Phys. Rev. Lett. 106, 113602 (2011).

[9] C. H. Bennett, F. Bessette, G. Brassard, L. Salvail, and J. Smolin, J. Cryptol. 5, 3 (1992).

[10] A. Muller, J. Breguet, and N. Gisin, Europhys. Lett. 23, 383 (1993).

[11] K. Mattle, H. Weinfurter, P. G. Kwiat, and A. Zeilinger, Phys. Rev. Lett. 76, 4656 (1996).

[12] D. Bouwmeester, J.-W. Pan, K. Mattle, M. Eibl, H. Weinfurter, and A. Zeilinger, Nature 390, 575 (1997).
[13] M. Barbieri, F. De Martini, G. Di Nepi, P. Mataloni, G. M. D'Ariano, and C. Macchiavello, Phys. Rev. Lett. 91, 227901 (2003).

[14] M. Rådmark, M. Żukowski, and M. Bourennane, New J. Phys. 11, 103016 (2009).

[15] K. J. Resch, K. L. Pregnell, R. Prevedel, A. Gilchrist, G. J. Pryde, J. L. O'Brien, and A. G. White, Phys. Rev. Lett. 98, 223601 (2007).

[16] P. B. Dixon, D. J. Starling, A. N. Jordan, and J. C. Howell, Phys. Rev. Lett. 102, 173601 (2009).

[17] P. Usachev, J. Söderholm, G. Björk, and A. Trifonov, Opt. Commun. 193, 161 (2001).

[18] D. N. Klyshko, Phys. Lett. A 163, 349 (1992).

[19] D. N. Klyshko, Sov. Phys. JETP 84, 1065 (1997).

[20] T. N. Thiele, Almindelig Iagttagelseslaere: Sandsynlighedsregning og mindste Kvadraters Methode (C. A. Reitzel, Copenhagen, 1889). Translated to English in Ann. Math. Statist. 2, 165 (1931).

[21] S. L. Lauritzen (ed.), Thiele: Pioneer in Statistics (Oxford University Press, New York, 2002).

[22] R. Kubo, J. Phys. Soc. Jpn. 17, 1100 (1962). 
[23] A. K. Jaiswal and C. L. Mehta, Phys. Rev. 186, 1355 (1969).

[24] T. Aoki and K. Sakurai, Phys. Rev. A 20, 1593 (1979).

[25] C. Brosseau, R. Barakat, and E. Rockower, Opt. Commun. 82, 204 (1991).

[26] W. Cai, M. Lax, and R. R. Alfano, Phys. Rev. E 63, 016606 (2000).

[27] W. Cai, X. Ni, S. K. Gayen, and R. R. Alfano, Phys. Rev. E 74, 056605 (2006).

[28] M. G. Raymer, A. C. Funk, and D. F. McAlister, in Quantum Communication, Computing, and Measurement 2, edited by P. Kumar, G. M. D’Ariano, and O. Hirota (Plenum, New York, 2000), p. 147.

[29] A. Luis and N. Korolkova, Phys. Rev. A 74, 043817 (2006).

[30] G. Björk, J. Söderholm, L. L. Sánchez-Soto, A. B. Klimov, I. Ghiu, P. Marian, and T. A. Marian, Opt. Commun. 283, 4440 (2010).
[31] E. Collett, Am. J. Phys. 38, 563 (1970).

[32] J. Schwinger, US Atomic Energy Commission Report No. NYO-3071, 1952 (unpublished); reprinted in Quantum Theory of Angular Momentum, edited by L. C. Biedenharn and H. van Dam (Academic, New York, 1965), p. 229.

[33] J. Söderholm, G. Björk, A. B. Klimov, L. L. Sánchez-Soto, and G. Leuchs (to be published).

[34] U. Schilling, J. von Zanthier, and G. S. Agarwal, Phys. Rev. A 81, 013826 (2010).

[35] R. Barakat, J. Opt. Soc. Am. A 6, 649 (1989).

[36] C. K. Hong, Z. Y. Ou, and L. Mandel, Phys. Rev. Lett. 59, 2044 (1987).

[37] O. Kwon, Y.-S. Ra, and Y.-H. Kim, Phys. Rev. A 81, 063801 (2010); Opt. Express 17, 13059 (2009).

[38] H. Prakash and N. Chandra, Phys. Rev. A 4, 796 (1971); G. S. Agarwal, Lett. Nuovo Cimento 1, 53 (1971). 